HU-EP-01/07

astro-ph/0102448

\title{
Gauge Invariant Perturbation Analysis for Quintessence with an Exponential Potential
}

\author{
Tassilo Ott \\ ott@physik.hu-berlin.de \\ Institut für Physik der Humboldt-Universität zu Berlin \\ Invalidenstraße 110 \\ D-10115 Berlin, Germany
}

\begin{abstract}
Gauge invariant adiabatic perturbations in the cosmology of a scalar field $\varphi(\vec{x}, t)$ gravitationally coupled to a perfect fluid (radiation or matter) are investigated. The potential of the scalar field is of exponential form. This model recently has received much attention in the context of 'quintessence' due to new observations of high redshift supernovae indicating an accelerating universe. The perturbations are treated in a gauge invariant formalism to systematically avoid the residual gauge modes in synchronous gauge. Two important attractor solutions of the unperturbed equations are investigated: the first corresponds to the case where the energy density of the unperturbed field $\rho_{(\varphi)}$ dominates the cosmology after a short time, the second to a solution with a time independent ratio $\rho_{(\varphi)} / \rho_{\text {hyd }}$. The main question addressed in this paper is if the inhomogenous fluctuations can be ignored as often argued or not. Indeed, in some important cases the perturbations do not die out with time meaning that the ratio of energy density of the inhomogenous fluctuations to the unperturbed part is constant after a while. Consequences for microwave background radiation and structure formation in the matter dominated era are considered in a straightforward manner and it is concluded that for similar initial values the anisotropy of the background radiation should be stronger in this model than just for radiation. Furthermore, structure formation should have happened slower than in standard cosmology.
\end{abstract}




\section{Introduction}

In this paper gauge invariant perturbations in the context of quintessence are investigated. Since Perlmutter et al. and Riess et al. [1, 2] have published their results that the universe is accelerating instead of decelerating, much work has been done to find the reason or at least a correct kinematic description for this behaviour. One of the best candidates is the so-called quintessence mattercomponent that effectively can be described by purely gravitational coupling of a scalar field to the standard matter (radiation or non-relativistic matter) [3]. There are many different suggestions for the form of the quintessence-potential (see for instance [3 6]). One of the first ones is the exponential potential as it was originally discussed by Wetterich [0 9] and Ratra and Peebles [10]. This potential will be investigated in the form:

$$
V(\varphi)=\hat{V} e^{-\frac{a}{M} \varphi} .
$$

In this definition $\mathrm{M}$ is the reduced planck mass $\left(M^{2}=M_{\mathrm{P}}^{2} / 16 \pi\right)$ and $a$ the only free model parameter. The amplitude $\hat{V}$ can be adjusted by a shift of the field $\varphi$ and therefore does not play an important role. This potential is not compatible with observation [11] which could be changed in late cosmology, but it still serves as a suitable toy model. The naturalness of such models has been discussed by Hebecker et al. [12]. Adiabatic Perturbations of this model in synchronous gauge were already discussed by Ferreira and Joyce [13, 14 and Amendola [15, 16] but the influence of inhomogenous perturbations onto the CMBR has been ignored.

\section{The "background" solutions}

From the Einstein equations, Energy-momentum conservation and the Klein-Gordon equation the three field equations for just time dependent quantities can be obtained for Robertson-Walker background [7]:

$$
\begin{aligned}
& \ddot{\varphi}(t)+3 H(t) \dot{\varphi}(t)+\frac{\partial V(\varphi)}{\partial \varphi}=0, \\
& \frac{1}{6 M^{2}}\left(V(\varphi)+\frac{1}{2} \dot{\varphi}^{2}(t)+\rho(t)\right)=H^{2}(t), \\
& \dot{\rho}(t)+3 H(t)(\rho(t)+p(t))=0 .
\end{aligned}
$$

This work is in an inflationary context, so the density parameter $\Omega \equiv \rho_{\text {tot }} / \rho_{\mathrm{C}}$ is set to 1 ; an extension to the other cases would be straightforward. An equation of state is needed for the perfect fluid component and therefore the convention

$$
3(\rho+p)=n \rho .
$$

will be used such that $n=4$ gives a radiation dominated and $n=3$ a matter dominated universe. The nonlinear equations admit two well-known "attractor solutions".

On the one hand this is the so-called "tracker solution" (which will be referred to as attractor solution I):

$$
\begin{aligned}
\bar{\varphi}(t) & =\bar{\varphi}_{0}+\frac{2 M}{a} \ln \frac{t}{t_{0}}, & H(t) & =\frac{2}{n t}, \\
\bar{\rho}(t) & =\frac{6 a^{2}-3 n}{a^{2}} M^{2} H^{2}(t), & V(t) & =\frac{6 n-n^{2}}{2 a^{2}} M^{2} H^{2}(t) .
\end{aligned}
$$


The name arises because the time dependence of the scalar field energy density tracks the behaviour of the perfect fluid. This solution exists (meaning it is reached after a short period for virtually all initial conditions) for $a^{2}>n / 2$ and there is no induced cosmological constant and consequently it does not allow for an accelerated expansion of the universe.

On the other hand there is the solution (which will be called attractor solution II):

$$
\begin{aligned}
\bar{\varphi}(t) & =\bar{\varphi}_{0}+\frac{2 M}{a} \ln \frac{t}{t_{0}}, & H(t) & =\frac{1}{a^{2} t}, \\
\bar{\rho}(t) & =\rho_{0}\left(\frac{t}{t_{0}}\right)^{-\frac{n}{a^{2}}}, & V(t) & =\frac{2 M^{2}}{a^{2}}\left(\frac{3}{a^{2}}-1\right) \frac{1}{t^{2}} .
\end{aligned}
$$

It corresponds to the case where $\bar{\rho}>>V$ and exists for $a^{2}<3$, but in the regime $3 / 2 \leq a<\sqrt{3}$ for $n=3$ the attractor solution I is dominant. The energy density part of a dynamical cosmological constant in this case is non-vanishing:

$$
\bar{\rho}_{\Lambda}=\frac{2 M^{2}}{a^{2} t^{2}}\left(\frac{3}{a^{2}}-\frac{6}{n}\right)
$$

Nucleosynthesis gives a constraint for $a$ during that period of $a>\sqrt{22} \approx 4.69$ [7] or $a>4.5-$ 5.5 [14. Therefore, a scenario seems to be appealing where $a$ is not fixed but decreasing with cosmic time. Then during radiation domination attractor solution I was valid with $a \sim 5$ at the time of nucleosynthesis. At the beginning of matter domination, attractor solution I still was valid, but recently the value of $a$ decreased below $a<\sqrt{3 / 2}$ and the universe "switched over" towards attractor solution II. This could explain the acceleration of the universe today through the effective cosmological constant.

\section{The gauge invariant perturbation equations}

The problem of residual gauge modes is known to cosmologists for a long time (see for instance [17]), but has attracted general attention in the early eighties when Brandenberger et al. [18] showed that indeed many of the generally agreed results on perturbation growth were not correct due to residual gauge modes. These cannot be eliminated in general within synchronous gauge. A sophisticated solution to this problem already had been offered by Bardeen [19] a few years earlier. He first took the approach to eliminate the gauge modes by making a transformation from the original variables to new ones that explicitly do not change under residual general coordinate transformations. This approach is taken in this paper, following Mukhanov et al. [20]. The most general scalar metric perturbations are parameterised by the four variables $\phi, \psi, B$ and $E$ within the line element

$$
d s^{2}=\left[(1+2 \phi) d t^{2}-R(t) 2 B_{\mid i} d x^{i} d t-\left[(1-2 \psi) \delta_{i j}+R^{2}(t) 2 E_{\mid i j}\right] d x^{i} d x^{j}\right] .
$$

Here the symbol $\mid$ denotes the covariant derivative on the hypersurface of constant time, $R$ is the scale factor and $t$ denotes cosmological time. Gauge invariant linear combinations of the so-called metric potentials $\phi$ and $\psi$ can be constructed by

$$
\begin{aligned}
& \Phi=\phi+\frac{\partial}{\partial t}[(B-R \dot{E}) R], \\
& \Psi=\psi-\dot{R}(B-R \dot{E}) .
\end{aligned}
$$


Gauge invariant scalar perturbation quantities for the perturbations $\chi, \partial \rho, \partial p$ of the field, the fluid energy density and the fluid pressure respectively, can be constructed analogously:

$$
\begin{array}{ll}
\varphi(t, \vec{x}):=\bar{\varphi}(t)+\chi(t, \vec{x}), & \chi^{(\mathrm{gi})}=\chi+R \dot{\bar{\varphi}}(B-R \dot{E}), \\
\rho(t, \vec{x}):=\bar{\rho}(t)+\delta \epsilon(t, \vec{x}), & \delta \epsilon^{(\mathrm{gi})}=\delta \epsilon+R \dot{\bar{\rho}}(B-R \dot{E}), \\
p(t, \vec{x}):=\bar{p}(t)+\delta \mathrm{p}(t, \vec{x}), & \delta \mathrm{p}^{(\mathrm{gi})}=\delta \mathrm{p}+R \dot{\bar{p}}(B-R \dot{E}) .
\end{array}
$$

The gauge invariant perturbation for a three vector like peculiar velocity becomes:

$$
u_{i}(t, \vec{x}):=\bar{u}_{i}(t)+\delta u_{i}(t, \vec{x}), \quad \delta u_{i}^{(\mathrm{gi})}=\delta u_{i}+R(B-R \dot{E})_{\mid i} .
$$

By using these quantities, it is possible to construct the gauge invariant Einstein equations:

$$
\begin{aligned}
& \Delta \Psi-3 \dot{R}(\dot{R} \Phi+R \dot{\Psi})+3 \mathcal{K} \Psi=\frac{R^{2}}{4 M^{2}} \delta T_{0}^{0}{ }^{(\mathrm{gi})} \\
& (\dot{R} \Phi+R \dot{\Psi})_{, i}=\frac{R^{2}}{4 M^{2}} \delta T_{i}^{0} \text { (gi) } \\
& {\left[\left(2 R \ddot{R}+\dot{R}^{2}\right) \Phi+R \dot{R} \dot{\Phi}+R^{2} \ddot{\Psi}+3 R \dot{R} \dot{\Psi}-\mathcal{K} \Psi+\frac{1}{2} \Delta D\right] \delta_{j}^{i}-\frac{1}{2} \gamma^{i k} D_{\mid k j}=-\frac{R^{2}}{4 M^{2}} \delta T_{j}^{i(\mathrm{gi})}} \\
& D:=(\Phi-\Psi) \quad \gamma_{i j}:=\delta_{i j}\left[1+\frac{1}{4} \mathcal{K}\left(x^{2}+y^{2}+z^{2}\right)\right]^{-2} .
\end{aligned}
$$

In this equation $\mathcal{K}$ denotes the spacial curvature in the Robertson-Walker metric. In the same manner a perturbed gauge invariant Energy-momentum tensor tensor can be derived. Since they couple only gravitationally, it is just the sum of fluid and scalar field components:

$$
\begin{aligned}
& \delta T_{0}^{0(\mathrm{gi})}=\delta \epsilon^{(\mathrm{gi})}-\dot{\bar{\varphi}}^{2} \Phi+\dot{\bar{\varphi}} \dot{\chi}^{(\mathrm{gi})}+V,_{\varphi} \chi^{(\mathrm{gi})} \\
& \delta T_{i}^{0 \text { (gi) }}=\frac{1}{R}(\bar{\rho}+\bar{p}) \delta u_{i}^{(\mathrm{gi})}+\frac{1}{R} \dot{\bar{\varphi}} \chi_{, i}^{(\mathrm{gi})} \\
& \delta T_{j}^{i(\mathrm{gi})}=\left(-\delta \mathrm{p}^{(\mathrm{gi})}+\dot{\bar{\varphi}}^{2} \Phi-\dot{\bar{\varphi}} \dot{\chi}^{(\mathrm{gi})}+V,_{\varphi} \chi^{(\mathrm{gi})}\right) \delta_{j}^{i} .
\end{aligned}
$$

For further discussions the curvature $\mathcal{K}$ is set to zero and it is possible to set $\Phi=\Psi$ because all spacial off-diagonal elements vanish in the perturbed Energy-momentum tensor. From (15) and (17) one gets an equation that gives a relation between $\Phi$ and $\chi^{(\mathrm{gi})}$ :

$$
\ddot{\Phi}+(n+1) H \dot{\Phi}+\left(n H^{2}+2 \dot{H}\right) \Phi+\frac{3-n}{3 R^{2}} \Delta \Phi=\frac{1}{4 M^{2}}\left(\frac{n-6}{3} \dot{\bar{\varphi}}^{2} \Phi+\frac{6-n}{3} \dot{\bar{\varphi}}^{(\mathrm{gi})}-\frac{n}{3} V_{, \varphi} \chi^{(\mathrm{gi})}\right) .
$$

This equation does not depend on $\delta \epsilon^{(\mathrm{gi})}, \delta \mathrm{p}^{(\mathrm{gi})}$ or $\delta u_{i}^{(\mathrm{gi})}$. This fact is quite remarkable, meaning that all dynamical information already is encoded in the two variables $\Phi$ and $\chi^{(\mathrm{gi})}$ and their derivatives. The fluctuation in the hydrodynamical part still can be obtained from the $(0,0)$-component of the Einstein equations.

In addition to this equation one needs the perturbation of the Klein-Gordon equation

$$
\varphi_{; \alpha}^{; \alpha}+V, \varphi=0
$$

in order to solve for the dynamics [21]:

$$
\ddot{\chi}^{(\mathrm{gi})}+3 H \dot{\chi}^{(\mathrm{gi})}-\frac{1}{R^{2}} \Delta \chi^{(\mathrm{gi})}+V_{, \varphi \varphi} \chi^{(\mathrm{gi})}-4 \dot{\bar{\varphi}} \dot{\Phi}+2 V_{, \varphi} \Phi=0 .
$$




\section{Discussion}

\subsection{Attractor solution I}

Equations (21) and (23) are getting fourier-transformed (denoted by ${ }^{\sim}$ ) and attractor-solution I is inserted. The equations are rescaled into logarithmic time $\tau:=\ln \left(t / t_{0}\right)$ and the equations are made dimensionless by the substitution $\tilde{\tilde{\chi}}:=\tilde{\chi} / M$. The two equations are of second order in time and it is possible to rewrite them as a system of first order matrix ODE's:

$$
\left(\begin{array}{c}
\tilde{\tilde{\chi}}^{(\mathrm{gi})} \\
\tilde{\tilde{\chi}}^{(\mathrm{gi}) \prime} \\
\tilde{\Phi} \\
\tilde{\Phi}^{\prime}
\end{array}\right)=\left(\begin{array}{cccc}
0 & 1 & 0 & 0 \\
\mathrm{f}_{1} & \mathrm{f}_{2} & \mathrm{f}_{3} & \mathrm{f}_{4} \\
0 & 0 & 0 & 1 \\
\mathrm{f}_{5} & \mathrm{f}_{6} & \mathrm{f}_{7} & \mathrm{f}_{8}
\end{array}\right)\left(\begin{array}{c}
\tilde{\tilde{\chi}}^{(\mathrm{gi})} \\
\tilde{\tilde{\chi}}^{(\mathrm{gi}) \prime} \\
\tilde{\Phi} \\
\tilde{\Phi}^{\prime}
\end{array}\right) \quad \text { with } \begin{cases}\mathrm{f}_{1}=\frac{2 n-12}{n}-\frac{k^{2}}{k_{\mathrm{R}}^{2}} e^{\left(2-\frac{4}{n}\right) \tau} & \mathrm{f}_{2}=\frac{n-6}{n} \\
\mathrm{f}_{3}=\frac{24-4 n}{a n} & \mathrm{f}_{4}=\frac{8}{a} \\
\mathrm{f}_{5}=\frac{6-n}{6 a} & \mathrm{f}_{6}=\frac{6-n}{6 a} \\
\mathrm{f}_{7}=\frac{n-6}{3 a^{2}}+\frac{(3-n) k^{2}}{3 k_{\mathrm{R}}^{2}} e^{\left(2-\frac{4}{n}\right) \tau} & \mathrm{f}_{8}=\frac{-n-2}{n}\end{cases}
$$

and $k_{\mathrm{R}}:=R_{0} t_{0}^{-1}$. The prime denotes the derivative with respect to logarithmic time. The perturbation of the hydrodynamical part can be obtained from the $(0,0)$-component of the Einstein equations:

$$
\frac{\tilde{\delta \epsilon}^{(\mathrm{gi})}}{\bar{\rho}}=\frac{6 n a-n^{2} a}{12 a^{2}-6 n} \tilde{\tilde{\chi}}^{(\mathrm{gi})}-\frac{n^{2} a}{12 a^{2}-6 n} \tilde{\tilde{\chi}}^{(\mathrm{gi}) \prime}+\left(\frac{n^{2}-12 a^{2}}{6 a^{2}-3 n}-\frac{n^{2} a^{2} k^{2}}{\left(6 a^{2}-3 n\right) k_{\mathrm{R}}^{2}} e^{\left(2-\frac{4}{n}\right) \tau}\right) \tilde{\Phi}-\frac{2 n a^{2}}{2 a^{2}-n} \tilde{\Phi}^{\prime} .
$$

It is common practice in cosmology to use the ensemble hypothesis for the universe (see for instance [22] or [23]) and so the $(4 \times 4)$-matrix needs to get rewritten as a $(10 \times 10)$-matrix for the correlation functions:

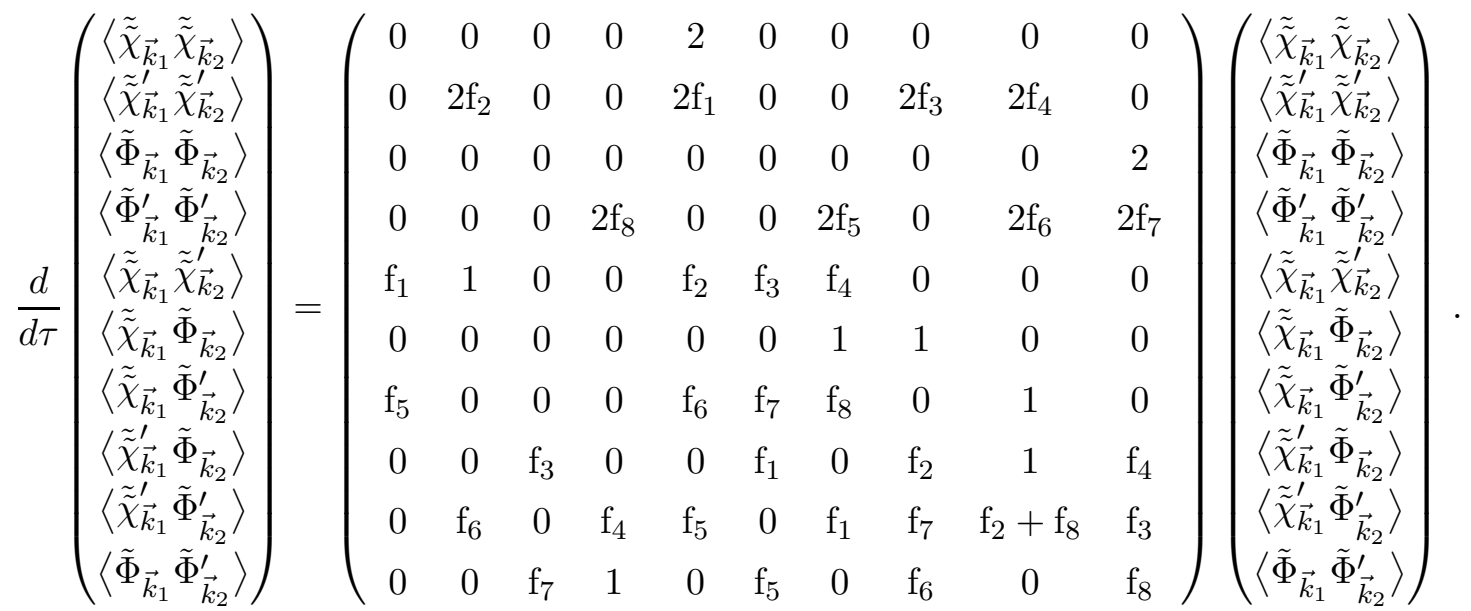

The energy density of the scalar field in second order can be separated into an unperturbed and a perturbed part:

$$
\begin{aligned}
\rho^{(\varphi)}(\dot{\varphi}, \varphi) & =\frac{1}{2} \dot{\varphi}^{2}+\frac{1}{2} R^{2}\left(\partial_{i} \varphi\right)^{2}+V(\varphi) \\
& =\underbrace{\frac{1}{2} \dot{\bar{\varphi}}^{2}}_{\bar{\rho}_{\text {kin }}^{(\varphi)}}+\underbrace{\dot{\bar{\varphi}} \dot{\chi}+\frac{1}{2} \dot{\chi}^{2}}_{:=\rho_{\text {kin,fluc }}^{(\varphi)}}+\underbrace{V(\bar{\varphi})}_{\bar{\rho}_{\mathrm{pot}}^{(\varphi)}}+\underbrace{V^{\prime}(\bar{\varphi}) \chi+\frac{1}{2} V^{\prime \prime}(\bar{\varphi}) \chi^{2}}_{:=\rho_{\mathrm{pot}, \text { fluc }}^{(\varphi)}} .
\end{aligned}
$$


In terms of correlation functions, this takes the form:

$$
\begin{aligned}
\left\langle\rho_{\text {kin,fluc }}^{(\varphi)}(\vec{x}, t)\right\rangle & =\frac{1}{2}\left\langle\dot{\chi}(\vec{x}, t) \dot{\chi}(\vec{x}, t)+\frac{1}{R^{2}} \partial_{i} \chi(\vec{x}, t) \partial_{i} \chi(\vec{x}, t)\right\rangle \\
& =\int_{-\infty}^{+\infty} \frac{d^{3} k}{(2 \pi)^{3}} e^{i \vec{k} \vec{x}} \int_{-\infty}^{+\infty} \frac{1}{2} \delta(\vec{k})\left[\langle\dot{\tilde{\chi}} \dot{\tilde{\chi}}\rangle(q, t)-\frac{1}{R^{2}} q_{i}\left(k_{i}-q_{i}\right)\langle\tilde{\chi} \tilde{\chi}\rangle(q, t)\right] \frac{d^{3} q}{(2 \pi)^{3}} \\
& =\frac{4 \pi}{(2 \pi)^{6}} \int_{0}^{\infty} \frac{1}{2}\left[\langle\dot{\tilde{\chi}} \dot{\tilde{\chi}}\rangle(q, t) q^{2}+\frac{1}{R^{2}}\langle\tilde{\chi} \tilde{\chi}\rangle(q, t) q^{4}\right] d q
\end{aligned}
$$

and

$$
\left\langle\rho_{\text {pot,fluc }}^{(\varphi)}(\vec{x}, t)\right\rangle=\frac{4 \pi}{(2 \pi)^{6}} \int_{0}^{\infty} \frac{1}{2} V^{\prime \prime}(\bar{\varphi})\langle\tilde{\chi} \tilde{\chi}\rangle(q, t) q^{2} d q .
$$

The assumption of gaussian random variables and therefore uncorrelated fourier modes was used. In order to do a numerical integration of the ODE's (26) one needs the initial conditions for every fourier mode. It is a prediction of inflation that the spectrum will be scale invariant (see for instance 23] or 24]), therefore the amplitude of every correlation function $k$-mode should be the same at the time the modes are crossing the horizon. For attractor solution I this does happen at

$$
\tau_{\mathrm{H}}=\ln \left(\sqrt[\frac{2}{n}]{\frac{k}{k_{\mathrm{R}} 2 \pi\left(1-\frac{2}{n}\right)}}\right) .
$$

The question how to choose the overall normalization of the spectra remains, and indeed one does not find a theoretical answer, because the underlying quantum theory is unknown. In this paper the main question is, how the fluctuations will qualitatively evolve in time. Therefore, the estimate from structure formation that the fluctuations of the radiation part compared to the background at the time of decoupling must have been of the order $10^{-3}$ should be sufficient [22]. For the sum of the correlation functions in $k$-space this corresponds to:

$$
\left\langle\frac{\delta \epsilon_{\mathrm{str}}(\vec{x}) \delta \epsilon_{\mathrm{str}}(\vec{x})}{\bar{\rho}_{\mathrm{str}}^{2}}\right\rangle=\frac{1}{\bar{\rho}_{\mathrm{str}}^{2}} \frac{4 \pi}{(2 \pi)^{6}} \int d q q^{2}\left\langle\tilde{\delta \epsilon}_{\mathrm{str}}(q) \tilde{\delta} \epsilon_{\mathrm{str}}(q)\right\rangle \approx 10^{-6} .
$$

This is taken as a normalization for the spectra. The fluctuations in non-relativistic matter and radiation at the time of decoupling are related by the simple equation:

$$
\left\langle\frac{\delta \epsilon_{\mathrm{mat}}(\vec{x}) \delta \epsilon_{\mathrm{mat}}(\vec{x})}{\bar{\rho}_{\mathrm{mat}}^{2}}\right\rangle=\left(\frac{3}{4}\right)^{2}\left\langle\frac{\delta \epsilon_{\mathrm{str}}(\vec{x}) \delta \epsilon_{\mathrm{str}}(\vec{x})}{\bar{\rho}_{\mathrm{str}}^{2}}\right\rangle .
$$

The result of the numerical integrations is shown in figure 1 for $a=2.5 .100$ different logarithmically distributed $k$-modes have been integrated from $\tau_{0}=0$ to $\tau=1$ with a fourth-order Runge-Kuttamethod using a stepsize of $\Delta \tau=0.01$ and a maximum $k$-value that corresponds to the horizon at $\tau_{0}$. The scale invariance has been implemented numerically.

It can be seen in the plots that during the radiation dominated epoch $(n=4)$ the perturbation in the kinetic energy density settles down to a constant value compared to the background whereas the perturbation of the potential energy dies out exponentially. This is an interesting result because it shows that during the radiation dominated epoch (if attractor solution I was valid) the perturbation of the scalar field cannot be neglected as often argued. For other values of $a$ the qualitative behaviour 

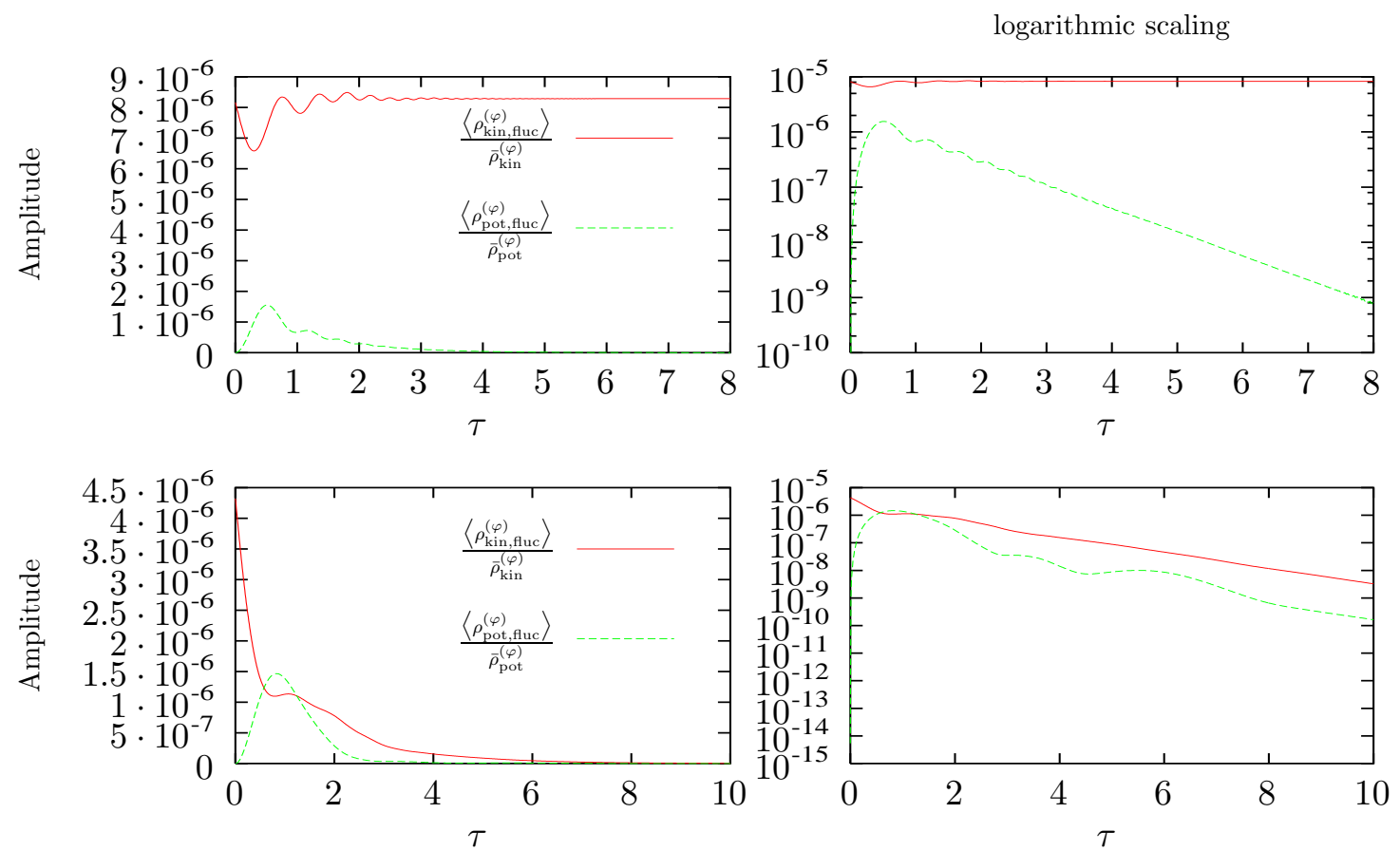

Figure 1: Ratios of kinetic and potential scalar field energy densities for attractor solution I with $a=2.5$ and $n=4$ (top), $n=3$ (bottom).

\begin{tabular}{|l||l|l|l|l|l|l|}
\hline$a$ & 1.414 & 2.0 & 2.5 & 4.0 & 5.0 & 10.0 \\
\hline$\frac{\left\langle\rho_{\text {kin,fluc }}^{(\varphi)}\right\rangle}{\bar{\rho}_{\text {kin }}^{(\varphi)}}$ & $1.41 \cdot 10^{-13}$ & $1.76 \cdot 10^{-6}$ & $8.28 \cdot 10^{-6}$ & $9.32 \cdot 10^{-5}$ & $2.54 \cdot 10^{-4}$ & $4.66 \cdot 10^{-3}$ \\
\hline
\end{tabular}

Table 1: Ratios of kinetic scalar field energy densities for attractor solution I.

is the same, but the value that the kinetic energy density ratio settles down to highly depends on $a$, as shown in table 1. For a value of $a=5$ as favored by nucleosynthesis one already gets $\left\langle\rho_{\text {kin,fluc }}^{(\varphi)}\right\rangle / \bar{\rho}_{\text {kin }}^{(\varphi)}=2.54 \cdot 10^{-4}$. The direct comparison of the fluctuation in the energy density of the scalar field perturbation and the radiation perturbation shows that this ratio is nearly $a$-independent and after some time takes on a value of $\left\langle\delta \rho_{\text {kin }}^{(\varphi)}(\vec{x}) \delta \rho_{\text {kin }}^{(\varphi)}(\vec{x})\right\rangle /\langle\delta \epsilon(\vec{x}) \delta \epsilon(\vec{x})\rangle \approx 1.7$. This means that the fluctuation in the kinetic part of the scalar field energy density is indeed greater than in the radiation energy density. Additionally, it is remarkable that after some time the scaling behaviour is the same for both (like for the background energy densities). The consequences for CMBR will be discussed later.

During the matter dominated epoch $(n=3)$ a different picture arises from the integrations: both kinetic and potential energy densities of the scalar field perturbation decay exponentially compared to the background. This means that even if the perturbation of the scalar field has been important during the radiation dominated epoch, in the matter dominated epoch it became negligible. Still there will be an interesting effect on the growing perturbation modes in the matter density. 


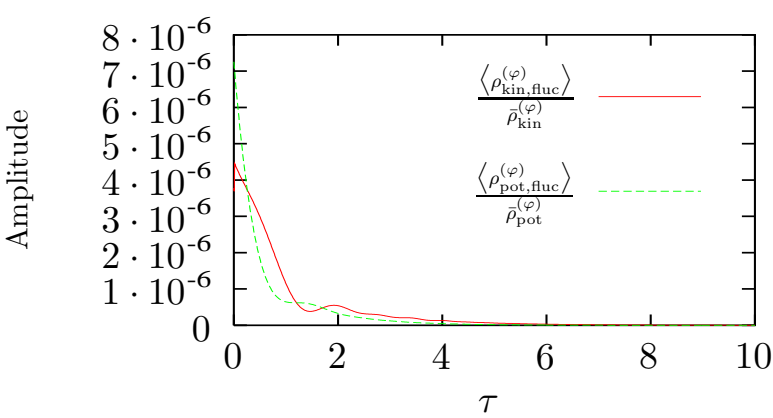

logarithmic scaling
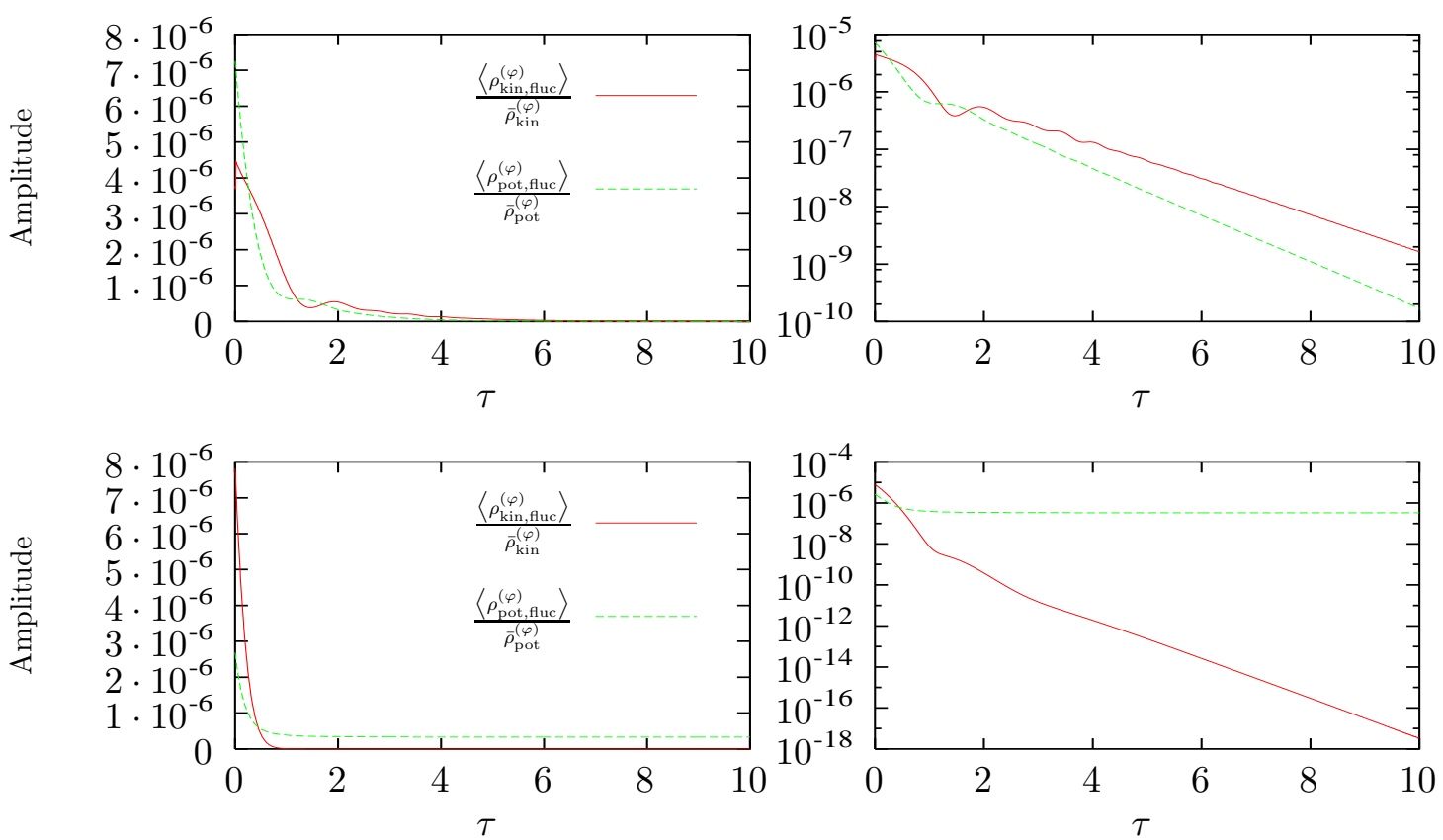

Figure 2: Ratios of kinetic and potential scalar field energy densities for attractor solution II with $a=1.2$ (top) and $a=0.8$ (bottom).

\subsection{Attractor solution II}

The gauge invariant perturbation equations can be written in an analogous form like (24) but with coefficients:

$$
\begin{aligned}
& \mathrm{f}_{1}=2-\frac{6}{a^{2}}-\frac{k^{2}}{k_{\mathrm{R}}^{2}} e^{\left(2-\frac{2}{a^{2}}\right) \tau}, \quad \mathrm{f}_{2}=1-\frac{3}{a^{2}}, \quad \mathrm{f}_{3}=\frac{12}{a^{3}}-\frac{4}{a}, \quad \mathrm{f}_{4}=\frac{8}{a}, \\
& \mathrm{f}_{7}=\frac{n}{3 a^{2}}-\frac{n}{a^{4}}-\frac{(n-3) k^{2}}{3 k_{\mathrm{R}}^{2}} e^{\left(2-\frac{2}{a^{2}}\right) \tau}, \quad \mathrm{f}_{6}=\frac{1}{a}-\frac{n}{6 a}, \quad \mathrm{f}_{5}=\frac{n}{2 a^{3}}-\frac{n}{6 a}, \quad \mathrm{f}_{8}=-\frac{n+1}{a^{2}}+1 .
\end{aligned}
$$

Furthermore, the equation for the time of horizon crossing is changing compared to (30):

$$
\tau_{\mathrm{H}}=\ln \left(\frac{1}{a^{2}} \sqrt[-1]{\frac{k}{k_{\mathrm{R}} 2 \pi\left(1-\frac{1}{a^{2}}\right)}}\right) .
$$

After these two alterations the equations can be integrated analogously and the energy density of the scalar field perturbations can be calculated. Attractor solution II becomes valid for $a<3 / 3$ and there is a qualitative difference in the behaviour for $a>1$ and $a<1$ (for $a=1$ the time dependence of the coefficients and therefore the $k$-dependence vanishes and this case is rather uninteresting). These two different cases are plotted exemplary for $a=1.2$ and $a=0.8$ in figure 2 . One sees that the ratios of the energy densities for $a>1$ decrease exponentially, while for $a<1$ this does happen just for the kinetic energy density ratio. The perturbation in the potential energy density of the scalar field settles down to a constant value. Naively, this might explain a part of the missing dark matter, as being stored in the local fluctuations of the scalar field. Attractor solution II could explain today's accelerated universe, as already mentioned. But there then must have been a transition from attractor solution I in recent times, meaning a long time after the beginning of matter domination. 


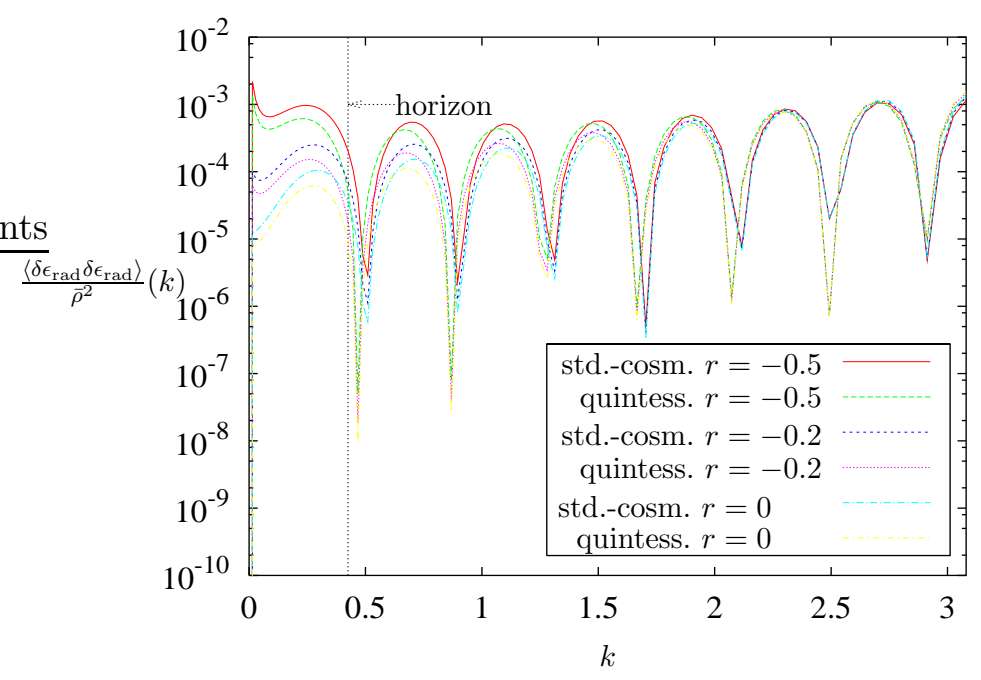

Figure 3: Comparison of the spectra of standard cosmology and the quintessential model with attractor solution I (normalized, $n=4, \tau=4, a=2.5$ )

During matter domination the ratio of the energy densities in the scalar field dies out exponentially. Therefore, the value of the potential energy density perturbation of the scalar field should be very small when attractor solution II becomes valid, and consequently the energy density stored in the perturbations is negligible.

\subsection{Possible Consequences for CMBR and structure formation}

The last scattering of the CMBR occurred approximately at decoupling. Therefore it should be generally possible to detect the effect of the scalar field perturbation from the fluctuation in the radiation part. It is instructive to directly compare standard cosmology with perfect fluid plus scalar field. The main problem is obvious: the initial normalization of the fluctuations has been chosen to approximately fit the fluctuations of the CMBR (31), so it would only be possible to detect a difference between the two models in the energy density if the qualitative behaviour of the radiation fluctuation ratio would be different. Therefore, also the case of standard cosmology has been examined in the "language" of the differential equations (24) and correlation functions (26). The result is that indeed one cannot recognize a qualitative difference between this and the case with scalar field and attractor solution I. This is rather discouraging but there is still the hope that there might be a difference in the $k$-spectra. To investigate this possibility the condition of scale invariance is given up at this time and different initial spectra are considered. Naive power spectra of the form

$$
P_{s_{i} s_{j}}(k, \tau) \propto k^{-\frac{r}{n}-1} e^{r \tau}
$$

are used, the free parameter is $r$. They are compared to the respective spectra without scalar field in figure 3. The difference between the two models is smaller than the difference between different initial spectra and is getting even smaller the earlier the modes have crossed the horizon to the inside (corresponding to greater values of $k$ ). The figure shows this for $\tau=4$, for increasing time the differences are also getting smaller. Therefore, one cannot distinguish the spectra of the fluctuation in the radiation density of the two models from these naive considerations. 


\begin{tabular}{|c|c|c|c|c|c|c|c|}
\hline$a$ & 1.414 & 2.0 & 2.5 & 4.0 & 5.0 & 10.0 & $\operatorname{Std} .-\operatorname{Cosmology}(a \rightarrow \infty)$ \\
\hline$y$ & 0.5478 & 0.9996 & 1.1282 & 1.2558 & 1.2838 & 1.3204 & 1.3324 \\
\hline
\end{tabular}

Table 2: Fitted growth exponent $y$ for matter density in matter dominated universe $(n=3)$, accuracy $\sim 10^{-3}$.

One also wants to consider the influence that the existence of the scalar field and its fluctuations have on structure formation. The root for structure formation is that during matter domination $(n=3)$ the fluctuations of the matter density are growing compared to the background. This is already known from standard cosmology, and it also can be seen in the integrations of this paper for $\varphi \equiv 0$, the growth of the correlation functions $\left\langle\delta \epsilon_{\text {mat }}(\vec{x}) \delta \epsilon_{\text {mat }}(\vec{x})\right\rangle / \bar{\rho}^{2}$ is proportional to $\exp (y \tau)$ with $y=4 / 3$ (for $\delta \epsilon_{\mathrm{mat}} / \bar{\rho}$ and normal time this is the familiar $t^{2 / 3}$ behaviour of the growing modes). In the model with scalar field and attractor solution I $(n=3)$ the growth exponent $y$ is changed. This change is $a$-dependent as shown in table 2. Therefore, the growth of the matter density fluctuations is smaller than in standard cosmology for all values of $a$ and which should lie in the region between $3 / 2$ and $\sim 5$ for structure formation as discussed earlier.

\section{Conclusions and Prospects}

There are two important cases where inhomogenous fluctuations cannot be ignored: first for attractor solution II and $a<1$ (which could play a role in late cosmology) and second in a radiation dominated universe plus quintessence with attractor solution I. The energy density ratio of kinetic scalar field fluctuations compared to the background settles down to a constant value, this numerical value highly depends on the model parameter $a$. As a consequence, the inhomogenous fluctuation in the radiation should have been stronger at the time of last scattering than without scalar field. Sadly, the initial normalization of the spectra is unknown and therefore, it is not possible to decide between the two models from such simple considerations. The form of the spectra also does not help very much. It might be useful to derive the spherical multipole expansion of the fluctuations on the one hand and to consider secondary anisotropies on the other. But this seems to make sense only if a more realistic description for matter and radiation is taken that pays more attention to the different particle species, see for instance [14, 25, 26] and a potential which is fully compatible with observation. Furthermore, it might be interesting to consider entropic perturbations in addition to the adiabatic perturbations. Appropriate Ansätze can be found in [20].

The growth exponent of the growing modes for structure formation in the matter dominated universe is changed by the coupling to scalar field perturbations. The growth is slower than in standard cosmology and the growth exponent highly depends on $a$. For realistic values the growth rate changes from $\exp (4 / 3 \tau)$ to between $\exp (1.28 \tau)$ for $a=5$ or $\exp (0.6 \tau)$ for $a=3 / 2$. This is a strong effect and it produces the unpleasant result that the structure formation should have taken a longer time for quintessence with exponential potential and the tracker solution. This seems to be a strong criteria to rule out or confirm quintessential models. Further consequences should be investigated for this model and also for other potentials.

\section{Acknowledgements}

I wish to thank C. Wetterich for useful discussions, helpful remarks and reading the manuscript and A. Hebecker for further prospects. The main part of this work has been done at Institut für 
Theoretische Physik, Philosophenweg 16, D-69120 Heidelberg. This work is supported by DFG.

\section{References}

[1] A. G. Riess et. al., Observational Evidence from Supernovae for an Accelerating Universe and a Cosmological Constant, Astron. J. 116 (1998) 1009-1038, astro-ph/9806396.

[2] Supernova Cosmology Project Collaboration, S. Perlmutter et. al., Discovery of a Supernova Explosion at Half the Age of the Universe and its Cosmological Implications, Nature 391 (1998) 51-54, astro-ph/9712212.

[3] R. R. Caldwell, R. Dave, and P. J. Steinhardt, Cosmological Imprint of an Energy Component with General Equation-of-State, Phys. Rev. Lett. 80 (1998) 1582-1585, astro-ph/9708069.

[4] I. Zlatev, L. min Wang, and P. J. Steinhardt, Quintessence, Cosmic Coincidence, and the Cosmological Constant, Phys. Rev. Lett. 82 (1999) 896, astro-ph/9807002.

[5] V. Sahni and L.-M. Wang, A new class of cosmological quintessence models, astro-ph/9910097.

[6] P. J. E. Peebles and A. Vilenkin, Quintessential inflation, Phys. Rev. D59 (1999) 063505, astro-ph/9810509.

[7] C. Wetterich, The Cosmon model for an asymptotically vanishing time dependent cosmological 'constant', hep-th/9408025.

[8] C. Wetterich, Cosmology and the Fate of Dilatation Symmetry, Nucl. Phys. B302 (1988) 668.

[9] C. Wetterich, Kaluza-Klein Cosmology and the Inflationary Universe, Nucl. Phys. B252 (1985) 309-320.

[10] B. Ratra and P. J. E. Peebles, Cosmological Consequences of a Rolling Homogenous Scalar Field, Phys. Rev. D37 (1988) 3406.

[11] T. Barreiro, E. J. Copeland, and N. J. Nunes, Quintessence arising from exponential potentials, astro-ph/9910214.

[12] A. Hebecker and C. Wetterich, Natural quintessence?, hep-ph/0008205.

[13] P. G. Ferreira and M. Joyce, Structure formation with a self-tuning scalar field, Phys. Rev. Lett. 79 (1997) 4740-4743, astro-ph/9707286.

[14] P. G. Ferreira and M. Joyce, Cosmology with a primordial scaling field, Phys. Rev. D58 (1998) 023503, astro-ph/9711102.

[15] L. Amendola, Perturbations in a coupled scalar field cosmology, astro-ph/9906073.

[16] L. Amendola, Coupled quintessence, astro-ph/9908023.

[17] S. Weinberg, Gravitation and Cosmology. John Wiley \& Sons, 1972.

[18] R. Brandenberger, R. Kahn, and W. H. Press, Cosmological Perturbations in the Early Universe, Phys. Rev. D28 (1983) 1809. 
[19] J. M. Bardeen, Gauge Invariant Cosmological Perturbations, Phys. Rev. D22 (1980) 1882.

[20] V. F. Mukhanov, H. A. Feldman, and R. H. Brandenberger, Theory of cosmological perturbations. Part 1. Classical perturbations, Phys. Rept. 215 (1992) 203-333.

[21] V. F. Mukhanov, Gravitational instability of the universe filled with a scalar field, Pis'ma Zh. Eksp. Teor. Fiz. 41 No.9 (1985) 402-405.

[22] J. A. Peacock, Cosmological Physics. Cambridge University Press, 1st ed., 1999.

[23] E. W. Kolb and M. S. Turner, The Early Universe. Addison-Wesley, 1st ed., 1990.

[24] P. Peebles, The Large-Scale Structure of the Universe. Princeton University Press, 1st ed., 1980.

[25] C.-P. Ma and E. Bertschinger, Cosmological perturbation theory in the synchronous and conformal Newtonian gauges, Astrophys. J. 455 (1995) 7-25, astro-ph/9506072.

[26] M. Doran, M. J. Lilley, J. Schwindt, and C. Wetterich, Quintessence and the separation of $C M B$ peaks, astro-ph/0012139. 